\title{
Urologic trauma and reconstruction: where are we today
}

\author{
Jairam R. Eswara ${ }^{1}$ \\ Published online: 8 April 2019 \\ (c) Springer-Verlag GmbH Germany, part of Springer Nature 2019
}

It is with great pleasure that we present our topical issue on Urologic Trauma and Reconstruction. We hope you find it stimulating and relevant. Trauma and reconstruction are broad fields requiring a varied skillset. As a result, my goal for this issue was to demonstrate the breadth of reconstructive urology, both in topic and geography. We were fortunate that so many world-renowned surgeons contributed to this effort.

Urethral stricture disease is a major topic to both the specialist and the general urologist. We have several outstanding manuscripts dealing with male urethral stricture disease by Drs. Kulkarni (India), Horiguchi (Japan), and Geminiani (Brazil). In addition, we have a valuable contribution on female urethroplasties from Dr. Lawrence (New Zealand).

In a related topic, Prof. Andrich (UK) shared the results of her new device to assist with difficult urethral catheterization. This exciting technology allows difficult catheterization without the need for cystoscopy.

A growing field within reconstructive urology is that of congenitalism or the care of young patients with congenital urological conditions as they enter adulthood. Drs. Agrawal and Wood from Cleveland Clinic (US) make a valuable contribution to the field with their manuscript on healthcare providers' perspectives in transitioning spina bifida patients.

Dr. Brandes (US) and his group present their results of a statewide analysis of urological trauma during hysterectomy.
Interestingly, they found a relatively low overall rate of urological injuries during hysterectomy, but the technique impacted the rate of injury and the type of repair necessary.

In many countries, urologic prosthetics constitute a major component of the reconstructive urologist's practice. Dr. Li (US) presents their large database analysis of penile prosthesis implantation and removal, while Prof. Fisch (Germany) describes her results of artificial urinary sphincter placement after buccal graft urethroplasty.

Finally, one of the world's experts in gender affirmation surgery and reconstructive urology, Dr. Djordjevic (Serbia), presented two excellent manuscripts. The first is an analysis of their results of patients who underwent latissimus dorsi flap phalloplasty for female to male transition. The second manuscript is regarding one of the more challenging entities for the reconstructive urologist, management of severe hypospadias.

Given its prevalence, urethral reconstruction is clearly a major part of genitourinary reconstruction. Reconstructive urology, however, is more than urethral surgery, and we have much to learn from our colleagues around the world. If we see ourselves as only urethral surgeons, we risk limiting ourselves, our impact, and our future. As a result, it is critical that we embrace the global nature of our field.
Jairam R. Eswara

jairam.eswara@steward.org

1 Saint Elizabeth's Medical Center, Tufts University School of Medicine, Boston, USA 